\title{
Estrategia didáctica para fortalecer las competencias de producción mediática desde la comunicación para el desarrollo
}

\author{
Teaching strategy to strengthen media production competencies \\ from the outset of communication for development
}

Mabel Andrea Navarrete Vega

Rosa María Romero González

\begin{abstract}
\begin{tabular}{l|l} 
RESUMEN & ABSTRACT
\end{tabular}
Con relación a la formación de los comunicadores, se parte de la convicción de que es necesario fortalecer sus competencias de producción mediática desde experiencias que les permitan comprender el proceso integral de producción desde una comunicación responsable, crítica y democrática. La metodología del estudio es exploratoria y cuantitativa, con la aplicación de una encuesta a estudiantes de Comunicación y Periodismo de la Universidad de Colima se buscó conocer sus percepciones sobre el nivel de competencias para desarrollar productos mediáticos desde la comunicación para el desarrollo. Los resultados reflejan que en la mayoría de los ítems sobre producción mediática y gestión de proyectos los encuestados no se consideran capaces de realizar la acción de forma autónoma. La información obtenida permite ofrecer una propuesta didáctica que fortalezca competencias vinculadas a la producción mediática como las habilidades digitales, de gestión de proyectos y responsabilidad social.

Palabras clave: enseñanza superior, comunicación y desarrollo, competencia mediática, tecnologías de información y comu-

Around the training of communicators, this study starts from the conviction that it is necessary to strengthen their media production skills from experiences that allow them to understand the integral production process from responsible, critical, and democratic communication. The methodology used is of an exploratory and quantitative nature, with the application of a survey to Communication and Journalism students at the Universidad de Colima, it was sought to know their perceptions on the level of competencies to develop media products from communication for development. The results reflect that, in most of the items on media production and project management, the respondents do not consider themselves capable of acting autonomously. The information obtained allows us to offer a didactic proposal that strengthens skills related to media production such as digital skills, project management, and social responsibility.

Keywords: higher education, communication and development, media competency, information and communication technologies.
\end{abstract} nicación.

Recibido: marzo 11 de 2021 | Aprobado: agosto 11 de 2021 | Publicado: septiembre 23 de 2021 DOI: https://doi.org/10.33010/ie_rie_rediech.v12i0.1208 


\section{INTRODUCCIÓN}

El nuevo escenario social traspasado por las tecnologías de información y comunicación (TIC) ha promovido cambios relevantes en los medios y en el vínculo del usuario con la información, lo que ha desencadenado en los últimos años la necesidad de desarrollar habilidades mediáticas e informacionales para promover la inclusión social a esta nueva realidad. Para la UNESCO (2011) dichas competencias tienen que ver con el conocimiento y comprensión sobre el funcionamiento, efectos y posibilidades de los medios y la información con el propósito de avanzar en ámbitos democráticos y de participación social en la población. Con este objetivo se establecieron, entre otros, directrices y marcos como la alfabetización mediática informacional (AMI) y el marco de competencias TIC para profesores (ICT-CFT) que incluyen ámbitos para el currículum e indicadores para el desarrollo de habilidades mediáticas, informacionales y digitales en estudiantes y docentes.

De forma paralela, en las últimas décadas estudios e investigadores han reflejado la necesidad de educar para los medios; de esta manera, la alfabetización mediática y digital en la población escolar ofrece posturas y enfoques que apuntan a fortalecer en los estudiantes el pensamiento crítico reflexivo, la creatividad y la autonomía personal frente al uso de estos recursos para ser coherentes con esta necesidad de alfabetizar en el uso de los medios (Grijalva y Urrea, 2017; Gutiérrez y Tyner, 2012; Singh, Grizzle, Yee y Culver, 2015; Valdivia, Herrera y Guerrero, 2015).

Tales propósitos de formación han conllevado la necesidad de identificar el nivel de competencias mediáticas en los estudiantes en sus diversos niveles educativos. De forma particular, sobre estudiantes universitarios en México se identifican estudios realizados por Grijalva y Lara (2019) y Grijalva y Moreno (2016) donde se discute, entre otros aspectos, la ausencia de contenidos de alfabetización mediática en los programas educativos en el país a pesar de las sugerencias de la UNESCO. Los resultados de

\footnotetext{
Mabel Andrea Navarrete Vega. Universidad de Colima, México. Doctorante de Innovación en Tecnología Educativa; responsable de gestión operativa de Agorante, grupo de investigación en sociedad y tecnología del Centro Universitario de Investigaciones Sociales de la Universidad de Colima; maestra en Tecnologías de Información. Coorganizadora de diferentes proyectos de ciencia básica y aplicada para diferentes instancias financiadoras; integradora de diferentes grupos de investigación y colaboradora con redes académicas nacionales e internacionales. Ha publicado diversos textos científicos y técnicos sobre temas de inclusión digital y tecnologías de información y comunicación. Correo electrónico: mnavarrete@ucol.mx. ID: https://orcid.org/ 00000002-5290-3127.

Rosa María Romero González. Profesora-investigadora en la Facultad de Informática de la Universidad Autónoma de Querétaro, México. Imparte asignaturas a nivel de licenciatura y en los programas de doctorado en Tecnología Educativa, doctorado en Innovación y Tecnologías Educativas, y doctorado en Ciencias de la Computación. Pertenece al Sistema Nacional de Investigadores, Nivel I, y cuenta con perfil ProDeP. Ha participado en diferentes proyectos de investigación, además de la publicación de artículos y capítulos de libros. También realiza direcciones de tesis de maestría y doctorado con temas relacionados con gestión del conocimientos y modelos educativos aplicando tecnologías de la información. Correo electrónico: rossyrg04@yahoo.com. mx. ID: https://orcid.org/0000-0002-7367-8299.
} 
los instrumentos aplicados en estas investigaciones denotan que existen diferencias entre los grupos de participantes sobre los niveles de competencia mediática, casi la mitad de los estudiantes encuestados de las áreas de ciencias sociales y humanidades no tienen dominio en la producción de contenido audiovisual y multimedia.

Estos autores, quienes han venido trabajando con este objeto de estudio desde hace más de una década, dejan entrever en sus discursos que la competencia mediática no es una habilidad espontánea en los universitarios y que requiere ser fortalecida desde una comprensión en la cual los medios son entendidos como constructores de la realidad e instrumentos que permiten la expresión libre y comprometida desde las diversas voces de la sociedad.

Por otra parte, una investigación centrada en estudiantes de periodismo de Colombia, Perú y Venezuela (Romero-Rodríguez y Aguaded, 2016) recupera resultados sobre competencias mediáticas para el consumo informativo; los encuestados aluden a que esta habilidad suelen adquirirla de forma autodidacta, pero también refieren la necesidad de contar con competencias mediáticas que les permitan enfrentar los medios con responsabilidad y capacidad crítica y reflexiva. De igual manera, un estudio realizado en 25 universidades españolas sobre el nivel de competencias mediáticas en universitarios (Aparicio, Tucho y Marfil, 2020) deja entrever que los jóvenes se autoperciben con altas capacidades mediáticas, sin embargo, al analizar sus respuestas se evidencia una limitada adquisición y comprensión sobre las dimensiones relacionadas a la competencia mediática. Cabe mencionar que en la búsqueda documental no se identificaron estudios sobre el nivel de competencias mediáticas en estudiantes del área de comunicación en México.

Sin embargo, se recuperan los resultados proporcionados por el Centro Nacional de Evaluación para la Educación Superior (CENEval) en el Examen General para el Egreso de la Licenciatura en Ciencias de la Comunicación (EGEL-Comunica) durante el año 2015, donde se identifica que el 48.3\% de los jóvenes de comunicación que presentaron el examen se encuentran en un nivel aún no satisfactorio con relación a las cinco áreas que componen el instrumento: publicidad, comunicación organizacional, investigación científica, productos comunicativos y periodismo. Si bien estos datos reflejan una situación preocupante sobre el nivel de competencias que adquieren los estudiantes durante su formación, resulta revelador que uno de los ejes fundamentales en la formación de un comunicador y que tiene relación con la producción mediática obtenga de igual manera resultados desfavorables: solo el $6.7 \%$ de los estudiantes se encuentra en un nivel sobresaliente y el $43.8 \%$ presenta un desempeño no satisfactorio.

Este problema es evidente en las capacidades de producción mediática evaluadas en los futuros comunicadores en México. Se considera además para este estudio si estos productos comunicativos se enmarcan dentro de los planteamientos que establece la 
UNESCO $(2009,2013)$ sobre la formación de comunicadores y periodistas. Estos discursos aluden a la necesidad de fortalecer el desarrollo del pensamiento crítico y analítico en estos profesionales más allá de las destrezas meramente técnicas, y que sus esfuerzos, ya sean de producción mediática u otra índole, reflejen un compromiso con la sociedad ante sus necesidades y problemáticas como agentes de cambio y de transformación social que aporten al fortalecimiento de una democracia sana y robusta.

En este sentido, es propósito de este estudio identificar las competencias vinculadas a la producción mediática en estudiantes universitarios de la carrera de Comunicación de una universidad en particular a través de la integración exprofeso de indicadores y lineamientos recomendados por especialistas en la materia y organismos como la UNESCO para proponer una estrategia didáctica que fortalezca las competencias evaluadas desde el marco de la comunicación para el desarrollo.

Este trabajo aporta a los estudios sobre la formación de comunicadores desde el enfoque de la comunicación para el desarrollo humano y el cambio social que trabajan investigadores de la Universidad de Colima y de la Universidad Autónoma de Aguascalientes. Un antecedente es el análisis de experiencias en el centro occidente de México publicado por Zermeño, Padilla y Fernández (2020).

El valor del estudio abona a esta línea de estudio y busca evidenciar la necesidad de una mayor apropiación en producción mediática por parte de los futuros comunicadores, así como el desarrollo de esta competencia desde un punto de vista más integral que el meramente técnico y desde los cánones de una comunicación que aporte al cambio social. Particularmente, brinda una propuesta práctica para su empleo didáctico como una oportunidad para ajustar y mejorar la práctica educativa ante el reto continuo de fortalecer la formación de nuestros futuros profesionales.

\section{Comunicación para el desarrollo}

La comunicación para el desarrollo - conocida como C4D por su traducción del inglés Communication for Development- es un campo teórico en el cual se prioriza el diálogo, la escucha, la confianza y la acción de compartir conocimientos y habilidades para debatir y aprender a fin de lograr cambios sostenidos y significativos en determinados grupos sociales. Para la División de América Latina y el Caribe de la Cooperación Suiza (COSUDE) la comunicación para el desarrollo busca impulsar

...la participación y el cambio social con los métodos e instrumentos de la comunicación interpersonal, medios comunitarios y tecnologías modernas de información. La C4D no es un añadido sino una actividad transversal en la gestión de proyectos para fortalecer el diálogo con socios y personas beneficiarias con el fin de aumentar la participación ciudadana y fomentar la apropiación y sostenibilidad [2014, p. 10)].

La trayectoria de la comunicación para el desarrollo se remonta hacia los años cincuentas del siglo pasado, a partir de investigaciones interesadas en el papel de los 
medios en el bienestar de las personas. Estos primeros postulados se enmarcaron en el paradigma de la modernización que surgió desde los países del norte y agencias del Banco Mundial para atender a países menos desarrollados. En este sentido, se asumían a los medios como un instrumento para modificar e instruir la mentalidad de los países subdesarrollados para acercarlos al ideal de desarrollo (Barranquero y Rosique, 2014).

De acuerdo con Rodríguez (2009), este discurso, comprendido como una comunicación vertical de los medios, fue cuestionado paulatinamente por los países afectados, dando pie a un paradigma de corte más participativo (de abajo hacia arriba) en el cual las acciones de comunicación surgen desde las comunidades y no para las comunidades. Resulta fundamental en los ejercicios de comunicación para el desarrollo la participación de los actores involucrados.

Particularmente en Latinoamérica, esta vertiente alternativa entendida como una práctica comunicativa transformadora da sus primeros pasos desde la comunicación pedagógica que buscaba reducir los niveles de alfabetismo en los años sesentas del siglo XX, pasando por los planteamientos de una educación liberadora y transformadora de Paulo Freire. Eventos como el Informe McBride de 1980 y el Word Congress Comunicación for Development del 2007 permitieron una mayor definición y comprensión general del campo de una comunicación encaminada a resolver problemas y promover el desarrollo humano, hasta los discursos de referentes más contemporáneos de la comunicación para el desarrollo como Pasquali (2007), Martín-Barbero (2011), Gumucio (2011) y Tufte (2015), por mencionar algunos.

El concepto de comunicación para el desarrollo y el cambio social se ha venido denominando de distintas formas, tales como comunicación alternativa, participativa, ecosocial, comunicación para la justicia social o comunicación para el cambio social. Si bien continúan debates en el ámbito académico sobre el alcance de cada término, de acuerdo con Beyoda (2015), todas estas referencias se conciben dentro del concepto global de la comunicación para el desarrollo, es decir, existe un hilo conductor que las une y apunta hacia una comunicación que busca generar transformaciones o cambios en las comunidades o agrupaciones desde sus propios referentes para avanzar en su desarrollo humano.

Este tipo de comunicación, a diferencia de la comunicación masiva, busca entablar un diálogo horizontal con los involucrados, promover la participación, facilitar el acceso a la información y el conocimiento, así como empoderar a las personas a través de una comunicación sensible al entorno cultural en una dinámica de colaboración en la cual se comparte y se aprende (COSUDE, 2014). Esta perspectiva se nutre de metodologías participativas como la investigación acción participativa (IAP) para realizar talleres, ejercicios de empoderamiento, radios comunitarias y producción audiovisual, cinematográfica y/o mediática en la que el aprendizaje se da de forma horizontal en los diferentes actores participantes (Mardones y Velásquez, 2015). 


\section{Competencias vinculadas a la producción mediática}

Las habilidades digitales se han posicionado para responder a los tiempos cambiantes y a una economía basada principalmente en la información y el conocimiento. Particularmente, para Scott (2015) la alfabetización digital se focaliza en el uso de capacidades cognitivas superiores para dar sentido a la información, los medios de comunicación y las tecnologías que se encuentran presentes en el contexto actual.

Este tipo de habilidades, las cuales en la literatura pueden aparecer como alfabetización digital, habilidades digitales, habilidades TIC, competencia digital, habilidades de internet, entre otros nombres, dejan entrever la complejidad del fenómeno y la velocidad con la que se modifican los elementos que competen a estas, así como los propósitos que persiguen. Sin embargo, de acuerdo con González (2012), se pueden identificar a partir de los años noventas dos posturas sobre alfabetización digital, una más focalizada en el ámbito tecnológico y otra más enfocada en los aspectos comunicativo, social y participativo. La primera apunta a una formación más técnica para el uso de herramientas tecnológicas y la otra, más presente en los últimos años, se relaciona con las posibilidades que otorgan estas tecnologías para el desarrollo de las personas.

En ese sentido, se asume a las TIC como un ámbito que resulta prioritario y que dialoga con otras habilidades que les permiten a las personas participar de una amplia gama de actividades, ya sean laborales, sociales o de ocio (Binkley, Erstad, Herman, Raizen, Ripley, Miller y Rumble, 2012; Van Laar, Van Deursen, Van Dijk y de Haan, 2020). Esta dinámica identifica un vínculo entre habilidades informacionales, mediáticas y digitales gracias a las posibilidades de la web 2.0 y la convergencia mediática y digital presentes en la vida cotidiana contemporánea (García, Gonzálvez y Aguaded, 2014; Grijalva y Urrea, 2017). De acuerdo a Trilling y Fadel (2009, citados en Scott, 2015, p. 7), “estas formas de alfabetización (...) están unidas por muchos vínculos. Una vez dominadas, estas formas de alfabetización, al igual que las formas tradicionales, permiten el dominio de otras competencias y habilidades necesarias para abrirse camino en el siglo XXI".

Especialmente en un estudiante de comunicación resulta prioritario este tipo de habilidades, sobre todo aquellas relacionadas con la producción mediática, asumida como un área primordial en los instrumentos de evaluación para egresados de las licenciaturas en Ciencias de la Comunicación a nivel país.

Dentro de este ámbito se reconocen las subáreas de preproducción, producción, postproducción y evaluación de productos comunicativos (CENEVAL, 2016), sin embargo, existen planteamientos de formación en que la producción mediática es más amplia y conlleva comprender, producir, difundir y evaluar procesos comunicativos, pero desde la participación del usuario como un actor de su propia narrativa (Soep, 2012). Este tipo de posibilidades son entendidas como la producción mediática activa, con la cual se fortalecen la comprensión e intervención en problemas sociales 
buscando reconocer la voz del actor social a través de un proceso de co-creación para promover una comunicación responsable, crítica y democrática (Tufte, 2015).

El concepto de productor mediático activo dialoga con el de comunicador-mediador, un postulado que plantea Martín-Barbero (2011) al visualizar al comunicador como un profesional que "asume como base de su acción las asimetrías y las desigualdades sociales y culturales, al participar como actor en la construcción de una sociedad democrática” (p. 18). Estos planteamientos son retomados en los trabajos de Zermeño, Padilla y Fernández (2020) en relación a la formación de comunicadores para el cambio social desde un producción mediática que busque atender la perspectiva del usuario en prácticas de la vida real.

Esto significa, en términos de Soep (2012), que:

En el proceso [de producción mediática activa] se examinan las implicaciones de los creadores que intentan ajustar el contenido a los usuarios en lugar de a las audiencias, y se plantean cuestiones que transforman los preceptos tradicionales de la alfabetización en los medios y sus prácticas. En la investigación sobre jóvenes y producción digital se cuestiona la idea que presenta Rushkoff del usuario como ser desventajado. En lugar de esto, se plantea al usuario como la fuerza que mueve la producción. Trabajar como productor, en otras palabras, requiere la habilidad de incorporar, ejecutar y estimular la posición del usuario. Lo que se obvia de alguna forma es hasta qué punto el usuario dirige la producción. Lejos de ser un carácter patético y marginal, el usuario emerge como árbitro ante los productos que se generan. Establecer la proximidad con el usuario y ser capaz de anticiparse y articular posibles puntos de vista son factores decisivos desde el punto de vista de la producción [p. 94].

Como productores mediáticos activos, los estudiantes abordan desafíos intelectuales y sociales interrelacionados. En tal sentido, Martin-Barbero (2011) precisa que el productor que se forma en las universidades no puede ser meramente un ejecutor, sino más bien un diseñador, alguien capaz de concebir el proceso en su totalidad, que va desde la idea, la realización como tal y el propósito social de dicho producto. Comprender la producción mediática desde esta mirada implica visualizarla como un proyecto en sí mismo, lo que se traduce en entender el tema abordado, trazar alcances y planes de acción, es decir, diseñar y gestionar un proyecto social.

En el marco de las competencias del siglo XXI, el enfoque del diseño de proyectos se identifica como una habilidad que se desarrolla dentro del ámbito de la colaboración (Binkley et al., 2012; Scott, 2015b; Wiek, Bernstein, Foley, Cohen, Forrest, Kuzdas, Kay y Keeler, 2016). En este contexto, las acciones relacionadas con la planeación, ejecución y seguimiento de un proyecto dialogan con el aprendizaje colaborativo, lo cual se produce al agrupar a los estudiantes de manera intencionada con el objeto de alcanzar una meta específica. Corresponde, más bien, a un acercamiento práctico al proceso de planeación, desarrollo, seguimiento y evaluación de un proyecto, más que al uso de metodologías y estándares establecidos desde el campo de la dirección administrativa de proyectos. 


\section{INDICADORES Y LINEAMIENTOS}

Los ítems considerados en el instrumento para medir las competencias vinculadas a la producción mediática se basan, por una parte, en los indicadores sobre competencia mediática establecidos por Pérez y Delgado (2012) y Ferrés y Piscitelli (2012), especialistas que han venido realizando estudios y proyectos en este campo por más de una década y que basan su trabajo desde el enfoque de la cultura participativa en los procesos comunicativos. Específicamente, se retoman los ámbitos relacionados con el proceso de producción mediática: diseño, desarrollo y difusión de un producto multimedia, así como el manejo de herramientas tecnológicas, alfabetización informacional y producción digital.

Dentro de este ámbito se añaden ítems sobre la vida activa de un producto mediático, la participación del usuario en el proceso y la atención a temas sociales relevantes, dimensiones que postula Soep (2012) desde el constructivismo social, en el cual el conocimiento se adquiere con otros en el desarrollo de contenidos digitales sobre problemáticas sociales asociadas a su impacto mediático. Estos aspectos dialogan con principios de la comunicación para el desarrollo sobre la importancia de la integración activa de los actores involucrados en las fases de identificación, desarrollo e implementación de estrategias de comunicación (Gumucio, 2011; Tufte y Mefalopulos, 2009).

Por otra parte, para la sección de gestión de proyectos en el instrumento aplicado se retoman los procesos sobre diseño, planeación, administración, seguimiento y evaluación de un proyecto, habilidad que en la actualidad resulta de utilidad tanto a nivel personal como profesional y/o académico (Ollé y Cerezuela, 2018). Así mismo los trabajos de Binkley et al. (2012) y Wiek et al. (2016), desde el marco de las competencias del siglo XXI, visualizan la gestión de proyectos desde el ámbito de la colaboración y el diálogo, lo cual armoniza con los lineamientos de la producción mediática activa al asumir al estudiante de comunicación en un rol de comunicador mediador que construye desde acciones que involucran el diseño, la ejecución y evaluación junto a otros hacia propósitos que aporten al cambio social.

Igualmente se consideran las directrices de la UNESCO sobre la formación para comunicadores y periodistas en sus documentos del Plan Modelo de Estudios de Periodismo (2009) y Model curricula for journalism education: A compendium of a new syllabi (2013), donde se ofrece información sobre el escenario y planes de estudio de las escuelas de comunicación en diversos países y se especifican aquellas competencias que se consideran relevantes en un profesional de la comunicación. En particular, sobre la producción mediática se postula el manejo hábil de herramientas para la edición, el diseño y la producción de material para medios de comunicación e información escritos, audiovisuales y en línea, desde una formación que propicie competencias para desenvolverse y aprovechar las herramientas que ofrece la sociedad y el cono- 
cimiento, así como la integración de una visión humanista como agente de cambio y transformador social que fomente y fortalezca la democracia en la sociedad.

\section{MÉTODo}

El texto refiere a una investigación de corte exploratorio y cuantitativo con la aplicación de una encuesta realizada a estudiantes de séptimo semestre y recién egresados de las carreras de Comunicación y Periodismo de la Universidad de Colima, con el propósito de conocer sus percepciones sobre el nivel de competencias para desarrollar productos mediáticos con TIC en contextos reales desde la comunicación para el desarrollo.

El cuestionario se aplicó a un total de 52 estudiantes de séptimo semestre y recién egresados de ambas licenciaturas, de edades entre 20 y 24 años, de los cuales el 56\% son mujeres y el 44\% hombres; el 69\% eran estudiantes de licenciatura en Comunicación y el 31\% de Periodismo. En cuanto a la condición de estudio de los participantes, el 67\% de los encuestados eran alumnos de séptimo semestre de ambas carreras y el 33\% recién egresados durante el 2019.

Se optó por esta población de estudio ya que se buscaba identificar si los estudiantes que estaban a punto de egresar y que habían tenido una suficiente formación en su disciplina, así como aquellos que acababan de insertarse al ámbito laboral, contaban con las competencias vinculadas a la producción mediática esperadas para un profesional del área de comunicación.

El instrumento fue estructurado de acuerdo con las tres variables principales: producción mediática, gestión de proyectos y habilidades digitales, con un total de 21 reactivos en escala de Likert; las opciones presentadas fueron: 1) Lo desconozco, 2) No soy capaz de hacerlo, 3) Sí, lo haría con ayuda, 4) Sí, lo haría sin ayuda, y 5) Sí, y sabría explicarlo. La sección de producción mediática incluyó ocho ítems relacionados con el desarrollo de productos comunicativos y experiencia en proyectos sobre problemáticas sociales. El apartado de gestión de proyectos contó con siete ítems cuyo fin fue reconocer el nivel de conocimiento de los procesos básicos de la gestión. Por su parte, los últimos seis ítems referían a habilidades digitales, en particular las informacionales y de producción digital. Este instrumento fue previamente sometido a una prueba piloto y validado por dos expertos en el área. El nivel de escala de Cronbach obtenido fue de 0.949 .

La recolección de datos se llevó a cabo durante el mes de agosto del 2019 en los espacios de la Facultad de Letras y Comunicación de la Universidad de Colima entre los alumnos de séptimo semestre, mientras que para los jóvenes recién egresados se optó por recomendación de los asesores pedagógicos de la Facultad, quienes les enviaron la invitación e instrumento vía correo electrónico. 
Para el análisis de los datos se utilizó el método estadístico descriptivo y de frecuencias, procedimiento que implicó establecer la distribución de frecuencia, es decir la cantidad de veces que aparece el valor asumido en cada categoría. Los datos se ubicaron en un cuadro de doble entrada para luego pasar los valores a términos relativos (porcentajes), así como la media o promedio de cada variable para identificar las medidas de tendencia central de los valores obtenidos (Rodríguez, 2009).

Ambas modalidades de la recolección de datos se ajustaron a los principios éticos y normas estipuladas para la investigación con personas. Los estudiantes fueron informados sobre el propósito y contexto de la encuesta, modalidad de aplicación, tiempo de realización, así como la condición de participar de forma voluntaria y anónima.

\section{Resultados}

La medición de las competencias vinculadas a la producción mediática en estudiantes que están por egresar y recién egresados de las licenciaturas de Comunicación y Periodismo arrojó un promedio general de $70 \%$, lo que indica un nivel intermedio de desarrollo.

De acuerdo con las opciones presentadas, se ubican en la categoría "pueden realizar la tarea, pero con ayuda", lo que evidencia poca capacidad de autonomía en los aspectos evaluados para alumnos de últimos semestres y recién egresados.

El menor nivel promedio fue de $64.6 \%$, obtenido en el ítem "Genero estrategias para dar seguimiento a la ejecución de un proyecto". El nivel más alto fue de 84.6\%, en el ítem "Edito, comparto y descargo distintos tipos de archivos en la nube". La tabla 1 presenta los promedios obtenidos luego del análisis de los datos recolectados. Cabe recordar que las categorías para cada ítem corresponden a: 1) Lo desconozco, 2) No soy capaz de hacerlo, 3) Sí, lo haría con ayuda, 4) Sí, lo haría sin ayuda, y 5) Sí, y sabría explicarlo.

Los promedios alcanzados para cada variable presentan diferencias: la media más elevada corresponde a la de habilidades digitales, con un promedio de $80 \%$, seguida por la de producción mediática con $70 \%$ de promedio. El menor nivel de desarrollo se obtuvo en la variable de gestión de proyectos, con 66\%.

\section{Producción mediática}

Se identifican las acciones de seguimiento de la vida activa de un producto mediático y estrategias de mejora como los ítems menos adquiridos de acuerdo con la percepción de los encuestados, con un promedio de $65.4 \%$, siendo los recién egresados quienes se declaran menos competentes con 55\%, y los estudiantes son más optimistas, obteniendo una media del 75\%. En contraparte, el promedio más alto en esta sección se obtiene, tanto en estudiantes como en los recién egresados, en la percepción de ser capaces de difundir un producto mediático, con un promedio de $82.6 \%$. 
Tabla 1. Porcentaje por escala de valoración y promedio de las variables.

\begin{tabular}{|c|c|c|c|c|c|c|}
\hline Ítems & 5 & 4 & 3 & 2 & 1 & Media \\
\hline \multicolumn{7}{|c|}{ Producción mediática } \\
\hline $\begin{array}{l}\text { 01. Comprendo cómo resolver problemáticas sociales desde la } \\
\text { comunicación }\end{array}$ & $19.2 \%$ & $25.0 \%$ & $42.3 \%$ & $13.5 \%$ & $0 \%$ & 3.5 \\
\hline $\begin{array}{l}\text { 02. Desarrollo proyectos que aborden una problemática social real y } \\
\text { significativa }\end{array}$ & $15.4 \%$ & $30.8 \%$ & $34.6 \%$ & $17.3 \%$ & $1.9 \%$ & 3.4 \\
\hline 03. Identifico y comprendo las etapas de la producción mediática & $5.8 \%$ & $34.6 \%$ & $50.0 \%$ & $5.8 \%$ & $3.8 \%$ & 3.3 \\
\hline 04. Realizo productos mediáticos & $23.1 \%$ & $44.2 \%$ & $28.8 \%$ & $0 \%$ & $3.8 \%$ & 3.8 \\
\hline $\begin{array}{l}\text { 05. Realizo productos mediáticos que integran la participación del } \\
\text { usuario en las distintas fases de la producción }\end{array}$ & $11.5 \%$ & $26.9 \%$ & $53.8 \%$ & $7.7 \%$ & $0 \%$ & 3.4 \\
\hline 06. Publico y difundo un producto mediático & $32.7 \%$ & $48.1 \%$ & $19.2 \%$ & $0 \%$ & $0 \%$ & 4.1 \\
\hline $\begin{array}{l}\text { 07. Diseño estrategias para dar seguimiento a la vida activa de un } \\
\text { producto mediático }\end{array}$ & $9.6 \%$ & $32.7 \%$ & $36.5 \%$ & $17.3 \%$ & $3.8 \%$ & 3.3 \\
\hline $\begin{array}{l}\text { 08. Genero una propuesta de mejora con base en el análisis de la } \\
\text { vida activa de un producto mediático }\end{array}$ & $13.5 \%$ & $23.1 \%$ & $44.2 \%$ & $15.4 \%$ & $3.8 \%$ & 3.3 \\
\hline
\end{tabular}

09. Defino un proyecto social pertinente, sustentado y factible de realizarse

\begin{tabular}{llllcl}
$11.5 \%$ & $30.8 \%$ & $40.4 \%$ & $17.3 \%$ & $0 \%$ & 3.4 \\
$15.4 \%$ & $34.6 \%$ & $28.8 \%$ & $5.8 \%$ & $15.4 \%$ & 3.3 \\
& & & & & \\
$13.5 \%$ & $42.3 \%$ & $38.5 \%$ & $5.8 \%$ & $0 \%$ & 3.6 \\
$17.3 \%$ & $26.9 \%$ & $38.5 \%$ & $13.5 \%$ & $3.8 \%$ & 3.4 \\
$15.4 \%$ & $26.9 \%$ & $34.6 \%$ & $19.2 \%$ & $3.8 \%$ & 3.3 \\
$15.4 \%$ & $26.9 \%$ & $28.8 \%$ & $23.1 \%$ & $5.8 \%$ & 3.2 \\
& & & & & \\
$9.6 \%$ & $30.8 \%$ & $38.5 \%$ & $17.3 \%$ & $3.8 \%$ & 3.3 \\
\hline
\end{tabular}

Habilidades digitales

16. Busco y selecciono diferentes fuentes de información valiosa en internet

$\begin{array}{llcccc}51.9 \% & 17.3 \% & 28.8 \% & 1.9 \% & 0 \% & 4.2 \\ 50.0 \% & 23.1 \% & 26.9 \% & 0 \% & 0 \% & 4.2 \\ & & & & & \\ 46.2 \% & 32.7 \% & 17.3 \% & 3.8 \% & 0 \% & 4.2 \\ 44.2 \% & 30.8 \% & 25.0 \% & 0 \% & 0 \% & 4.2 \\ & & & & & \\ 28.8 \% & 40.4 \% & 26.9 \% & 3.8 \% & 0 \% & 3.9 \\ 30.8 \% & 30.8 \% & 13.5 \% & 17.3 \% & 7.7 \% & 3.6\end{array}$


Cabe mencionar que si bien estos promedios se ubican en un nivel intermedio, se evidencia que en todos los ítems evaluados en producción mediática ni el 50\% de los encuestados se consideran capaces de realizar la acción de forma autónoma y mucho menos de saber explicarla. Estos resultados sobre el bajo manejo del proceso de producción para estudiantes por egresar o recién egresados coinciden con los resultados del EGEL-Comunica (2015), en los cuales el 43\% de sujetos evaluados del área de Comunicación se ubica en un nivel de desempeño no satisfactorio en el área de productos comunicativos que engloban los procesos de preproducción, producción y postproducción.

\section{Gestión de proyectos}

La identificación de las fases de gestión, el seguimiento y la elaboración de informes obtuvieron los promedios más bajos, al alcanzar un promedio de 64 y 66 por ciento respectivamente. Estos promedios menores se presentan tanto en estudiantes como en los recién egresados, a excepción del indicador sobre reconocer las fases de gestión de proyectos, en el que los estudiantes se ubican 20\% más capaces en relación a los recién egresados.

Por su parte, el valor más alto en ambos grupos se encontró en la capacidad de redactar objetivos de un proyecto, con un promedio del $72.6 \%$.

La distribución de los porcentajes por categorías se presentan más dispersa en este apartado y con mayor presencia de las categorías "lo desconozco" o "no soy capaz de hacerlo", si se compara con las otras dos secciones del instrumento. Tal situación se debe probablemente a que estos contenidos no son propios de la formación en comunicación, sin embargo se reconoce el trabajo de proyectos como técnica de enseñanza en la formación universitaria (ANUIES, 2011), y en particular el documento sobre la formación de comunicadores y periodistas de UNESCO (2009) sugiere que los jóvenes comunicadores cuenten con oportunidades educativas que le permitan desarrollar habilidades para organizar y planificar la actividad comunicativa.

\section{Habilidades digitales}

A diferencia de las secciones anteriores, este apartado presenta los mayores porcentajes en las categorías de "soy capaz de realizarlo sin ayuda" y "puedo explicarlo". La mayoría de los ítems del apartado obtienen promedios en el rango del 80\%, a excepción del indicador sobre la capacidad para reconocer las posibilidades de las TIC en la solución de problemáticas sociales, con $72 \%$ de promedio. Esta capacidad es la menos apropiada en esta sección, tanto por los estudiantes, con $81 \%$, como por los recién egresados, con $62 \%$.

Estos resultados coinciden con investigaciones donde los jóvenes se perciben bastante competentes en la mayoría de los ámbitos digitales (Bordas y Arras, 2018; 
Cabezas, Casillas, Sanches y Teixeira, 2017), sin embargo, es preciso señalar que estudios de mayor profundidad contradicen esta percepción aludiendo a que existe poca evidencia empírica de que los jóvenes sean hábiles para dar un uso efectivo a estas tecnologías (Acosta, 2017; Castellanos, Sánchez y Calderero, 2017).

\section{Condición de estudio}

En cuanto a los resultados que no son objeto de este estudio pero que se obtienen como parte del análisis, cabe mencionar que no se identifican diferencias significativas por género o por tipo de licenciatura (Comunicación y Periodismo) en las variables evaluadas, no obstante, la condición de estudio entre estudiantes y recién egresados sí denota diferencia en los resultados. La tabla 2 presenta los porcentajes por categorías, mismas que reflejan que los estudiantes se autoperciben con mayores capacidades en comparación a los jóvenes recién egresados en las tres variables observadas. Es probable que las exigencias del escenario laboral le permitan al recién egresado reconocer de forma objetiva el nivel de las habilidades con las que cuenta.

Los resultados reflejan que en la mayoría de los ítems sobre producción mediática y gestión de proyectos los encuestados no se consideran capaces de realizar la acción de forma autónoma, pero gran parte de ellos se asumen con posibilidades de ejecutar la acción si cuentan con la ayuda o guía para realizarlo. La orientación constante del docente para realizar las tareas puede ser una limitante, aunque esto también se denota en los recién egresados. En síntesis, la información obtenida puede interpretarse como una falta de apropiación de dichas habilidades, lo que hace un llamado para establecer acciones didácticas dentro del proceso de enseñanza que fortalezcan estos aprendizajes considerados relevantes en la formación de un comunicador.

\section{Propuesta de producción mediática aCtiva}

Se propone un modelo de producción mediática activa para fortalecer competencias digitales sobre gestión de proyectos y responsabilidad social en estudiantes de comunicación, en el cual profesores y especialistas acompañen los procesos creativos de

Tabla 2. Frecuencias por tipo de competencias evaluadas por condición de estudio.

\begin{tabular}{|c|c|c|c|c|c|c|}
\hline \multirow[b]{2}{*}{ Escala } & \multicolumn{2}{|c|}{ Producción mediática } & \multicolumn{2}{|c|}{ Gestión de proyectos } & \multicolumn{2}{|c|}{ Habilidades digitales } \\
\hline & Estud. & $\overline{\text { Egres. }}$ & Estud. & $\overline{\text { Egres. }}$ & Estud. & Egres. \\
\hline Sí, y sabría explicarlo & $11.4 \%$ & $0 \%$ & $14.3 \%$ & $5.9 \%$ & $42.9 \%$ & $23.5 \%$ \\
\hline Sí, lo haría sin ayuda & $34.3 \%$ & $11.8 \%$ & $45.7 \%$ & $11.8 \%$ & $45.7 \%$ & $11.8 \%$ \\
\hline Sí, lo haría con ayuda & $54.3 \%$ & $47.1 \%$ & $40.0 \%$ & $23.5 \%$ & $11.4 \%$ & $64.7 \%$ \\
\hline No soy capaz de hacerlo & - & $41.2 \%$ & - & $47.1 \%$ & - & - \\
\hline Lo desconozco & - & - & - & $11.8 \%$ & - & - \\
\hline
\end{tabular}


comunicación de los educandos en un trabajo basado en proyectos con actores sociales, articulando procesos de formación, asesorías, prácticas comunicativas, inclusión digital y participación social sobre una dinámica de aprendizaje con otros y para otros.

Esta propuesta dialoga con la experiencia formativa "Media Lab para la producción mediática activa” descrita en la publicación de Zermeño, Padilla y Fernández (2020), coincidiendo en el propósito de ofrecer a los estudiantes un escenario para un aprendizaje significativo y constructivista, siendo ellos los protagonistas de sus propios aprendizajes. Lo que se busca es ofrecer entornos de aprendizaje que les propicien adaptar e integrar nuevos conocimientos a través de la práctica. Entre estas estrategias se encuentra el desarrollo de proyectos en los que intervienen la participación y la resolución de problemas en contextos de la vida real, lo que se constituye en una sólida acción de aprendizaje para el desarrollo de competencias del siglo XXI (Prasad, Raj y Bagale, 2017; Scott, 2015b).

En este contexto, se asume la definición de estrategia didáctica propuesta por González y Triviño (2018), entendida como:

El conjunto de procedimientos o recursos, utilizados por los docentes, apoyados en técnicas de enseñanza, que tienen por objeto llevar a buen término la acción didáctica, es decir, alcanzar los objetivos de aprendizaje, un aprendizaje que se entiende significativo mejorando la calidad del proceso [p. 373].

Durante la ejecución se propone la estrategia de trabajo por proyectos para propiciar la relación entre estudiantes y beneficiarios, en la cual se rescate la voz de estos últimos a través de procesos de co-creación. Se busca desde esta estrategia que se desarrollen diferentes capacidades en los estudiantes para que se asuman como productores mediáticos activos, acompañados por el docente y asesores. Desde la revisión de literatura se comprende que, en las experiencias basadas en el desarrollo de proyectos con actores sociales en contextos reales, el estudiante puede obtener una mejor comprensión de las problemáticas sociales y dar pie al desarrollo de soluciones creativas de manera ética, creativa y reflexiva con el apoyo de diversos recursos, incluyendo las TIC.

De igual forma, la producción mediática activa comprende que los procesos de enseñanza-aprendizaje estén distribuidos y diversificados. Docente y especialistas acompañan los procesos creativos de comunicación de los estudiantes al trabajar con beneficiarios reales, constituyéndose el modelo como una red que, desde la teoría del actor red (ANT), integra la interrelación entre diferentes actantes en igualdad de condiciones (Latour, 2008).

La fase de producción mediática activa está compuesta por cinco momentos: construcción de conocimiento, análisis de necesidades, diseño y planeación, producción y seguimiento, y evaluación (figura 1). Cabe mencionar que, si bien se propone desarrollar los momentos de forma consecutiva, existen ciertos momentos que se 
solapan, como por ejemplo análisis y diseño, y otros que se presentan durante todo el proceso de la producción mediática, como el seguimiento y evaluación.

- En un primer momento se lleva a cabo la construcción de conocimientos en los estudiantes sobre temas necesarios para realizar los proyectos específicos: problemáticas sociales, diseño y gestión de proyectos, producción mediática basada en el rol de comunicador-mediador, y posibilidades y alcance de las TIC. En este sentido, se busca ofrecer un panorama sobre el papel de la comunicación en el desarrollo social y humano, rescatando el papel de los usuarios dentro del proceso de la producción mediática.

Los jóvenes, en conjunto con el docente, valoran y definen temáticas de interés por equipo, buscando que los temas sean de alguna problemática social del entorno local. Así también se explora la viabilidad de acercamiento a especialistas de acuerdo al tema seleccionado.

De igual manera, durante esta fase participan asesores de contenido en una modalidad tipo foro dentro del salón de clase, en la cual los estudiantes a través de preguntas obtienen información de la situación de los problemas sociales previamente revisados en la clase, así como un acercamiento a las condiciones del fenómeno en el escenario real.

- La segunda parte corresponde al análisis de necesidades, con el cual se busca establecer una estrategia para realizar una aproximación al tema, localizando y analizando información, así como organismos o actores vinculados y, en particular, el actor social (beneficiario) que vivencia la problemática, para que sea la voz de los involucrados directos en el contenido que se producirá. En este punto resulta relevante instruir y orientar al estudiante en la identificación preliminar del problema para luego ajustarlo según la interacción con el beneficiario al dialogar sobre su realidad, necesidades y expectativas. Otro aspecto que resulta clave en este momento es orientar a los estudiantes para lograr un acuerdo verbal con el beneficiario sobre la modalidad de trabajo del proyecto, la que implicará tiempos para la colaboración conjunta y la disposición activa de participar.

- Posteriormente se presenta la tercera fase del modelo, que conlleva elaborar el plan de trabajo por parte de cada equipo; aquí se definen las diversas etapas del trabajo, actividades, tiempos, roles y recursos necesarios para la ejecución. Se comparten los planes generados entre los equipos, el docente y en particular con el beneficiario del proyecto para dar paso a retroalimentación, posibles ajustes y mejoras.

- Como parte del cuarto momento del modelo se ejecutan los proyectos por equipo. En esta fase se realizan las distintas actividades planeadas, así como el seguimiento cercano del docente para llevar a cabo el trabajo acordado y resolver en conjunto con los estudiantes las eventuales situaciones que puedan presentarse 


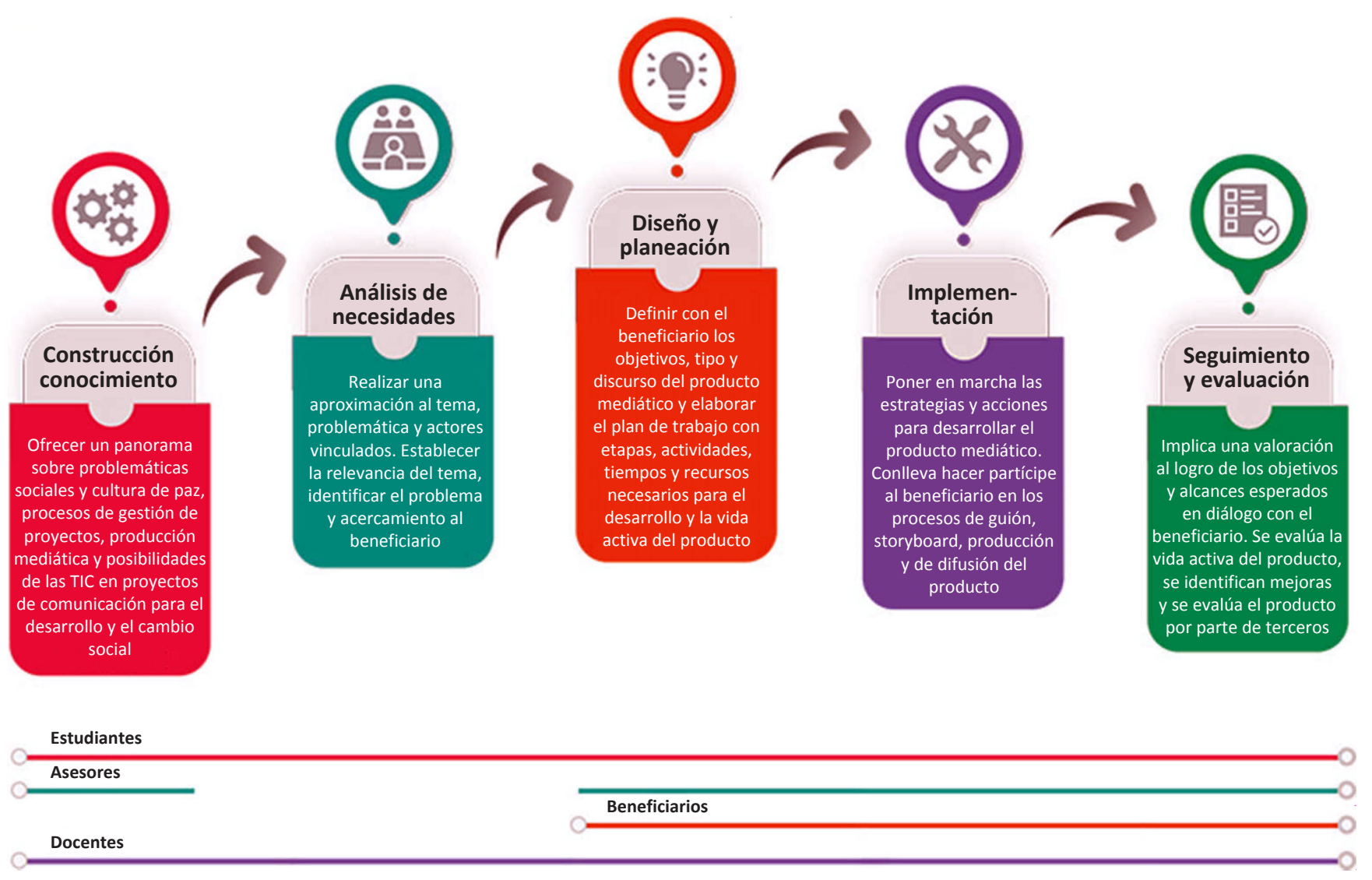

Figura 1. Fases de la propuesta del modelo de producción mediática.

Fuente: Construcción personal.

o el reajuste del plan de trabajo. Igualmente, en este periodo se abre la posibilidad de establecer contacto con asesores de contenido para orientar a los jóvenes sobre ciertos marcos o referencias que les permitan una mejor descripción de la problemática social, así también con asesores de producción mediática y digital que les guíen al resolver dudas técnicas o relacionadas con el desarrollo. Específicamente, este momento consiste en la generación de contenidos digitales (spots, infografías, videos, podcasts, animaciones, blogs, etc.) acordes a las necesidades de comunicación del beneficiario del proyecto. Este trabajo de producción consiste en la búsqueda de información, características del producto digital, generación del guión, diseño y producción. La participación del beneficiario durante estas actividades resulta crucial para generar un producto mediático acorde a sus necesidades de comunicación.

- Finalmente, los productos mediáticos se disponen en una plataforma digital. Los equipos diseñan una estrategia para la divulgación en redes sociales, lo que supone desarrollar productos asociados y obtener el aval de los beneficiarios para que dicha estrategia se ejecute o se ajuste. La evaluación del producto mediático se 
plantea a través del seguimiento de las métricas que automáticamente generan las redes sociales por un periodo determinado, dando la medida de la vida activa de este. Paralelamente, el beneficiario realiza una evaluación del producto mediático generado por los estudiantes. Con esta información se busca conocer su alcance y apreciaciones para reflexionar en torno a los resultados y establecer posibles mejoras.

Finalmente, se propone buscar la posibilidad de realizar una presentación entre pares académicos, beneficiarios y estudiantes para divulgar el trabajo realizado y exponer la experiencia obtenida.

\section{CONClusiones}

De acuerdo con los resultados expuestos, es necesario establecer estrategias didácticas que fortalezcan las competencias vinculadas a la producción mediática en la formación del comunicador. Esta acción educativa no considera como elemento central el desarrollo de habilidades técnicas para la producción, sino que avanza sobre una perspectiva crítica en la cual el productor se vuelve un mediador desde el compromiso social. Esto implica recuperar la voz y la participación del actor social en un proceso de co-producción, en diálogo y comprensión de esta realidad, para generar un mensaje comunicativo responsable, democrático y, ¿ ¿por qué no?, también estético.

Resulta relevante proponer un modelo práctico para la producción mediática desde la perspectiva de la comunicación para el desarrollo como un recurso que puede ser aplicado en las aulas. Esta propuesta pretende avanzar en ese propósito, así también generar conocimiento en este ámbito, ya que si bien existen diversas experiencias de trabajo de campo, las cuales pueden tener intenciones loables, se denota una ausencia en sistematizar de forma metódica dichas acciones. La ausencia de indicadores, marcos conceptuales o estudios comparativos sobre la producción mediática en comunicadores en la literatura científica explorada evidencia esta necesidad.

Por otra parte, de acuerdo a los resultados se pueden notar diferencias entre las percepciones de los estudiantes y los recién egresados sobre el nivel de capacidades vinculadas a la producción mediática con las que cuentan. Este hallazgo requiere de mayores estudios para su confirmación, no obstante, permite hacer un llamado a las universidades para implementar y/o reforzar estrategias educativas en las que los estudiantes puedan interactuar en escenarios reales y así obtener oportunidades de probarse y ajustar sus percepciones sobre los niveles de conocimientos adquiridos.

Cabe añadir que el modelo expuesto presenta determinadas fases para su ejecución, sin embargo, esta propuesta demanda aspectos elementales como, por ejemplo, que los estudiantes que participan cuenten con ciertos conocimientos de la disciplina, sobre todo de habilidades informacionales, de gestión de la información y de producción multimedia. De igual forma requiere la disposición, la actitud de participar 
y el apoyo de varios actores: estudiantes, actor social, asesores, dirección, entre otros. También es necesario que el docente asuma de forma consciente y responsable que este tipo de estrategias demanda mayor tiempo de diseño, realizar diversas acciones de gestión y otorgar un acompañamiento continuo a los estudiantes en el proceso.

Una de las limitantes de este estudio tiene que ver con la muestra. La encuesta se centra en un grupo específico de 52 estudiantes de una universidad del centro de México, no se aplicó el instrumento en otros lugares, por lo tanto, sus resultados son situados en un contexto educativo y cultura particular. Otra limitante es que solamente fue un acercamiento cuantitativo, reduciendo las posibilidades de una mayor profundidad de observación. A pesar de estas limitaciones, los datos de este estudio y su propuesta de estrategia didáctica podrían ser tomados en consideración tanto en la formación del alumnado de las carreras de Comunicación como en el posible diseño y evaluación de programas específicos dirigidos a la mejora de la producción mediática.

Algunas posibilidades de estudios posteriores que surgen a partir de este trabajo incluirían replicar la aplicación de la encuesta a una muestra más amplia, implementar la propuesta y dar seguimiento a su ejecución de forma sistemática; de igual manera, realizar comparaciones de experiencias en distintos contextos, ya que al trabajar con grupos sociales para la producción mediática desde la comunicación para el desarrollo se asume que su aplicación no es mecánica, sino que cada experiencia al ejecutarse variará y ofrecerá resultados distintos por las particularidades del entorno donde se aplique, ofreciendo mayor riqueza de información que fortalecerá al modelo y por ende a la formación de la producción mediática activa.

\section{REFERENCIAS}

Acosta, D. (2017). Tras las competencias de los nativos digitales: avances de una metasíntesis. Revista Latinoamericana de Ciencias Sociales, Niñez y Juventud, 15(1), 471-489. https://doi.org/10.11600/169271 5x.1513014062016 471.

ANUIES [Asociación Nacional de Universidades e Instituciones de Educación Superior] (2011). Innovación curricular en instituciones de educación superior: pautas y procesos para su diseño y gestión. México: ANUIES.

Aparicio, D., Tucho, F., y Marfil, R. (2020). Las dimensiones de la competencia mediática en estudiantes universitarios españoles. Icono14, 18(2), 217-244. https://doi.org/10.7195/ri14.v18i2.1492.

Barranquero, A., y Rosique, G. (2014). La formación en comunicación/educación para el cambio social en la universidad española. Rutas para un diálogo interdisciplinar. Cuadernos.info, (35), 83-102. https:/ / doi.org/10.7764/cdi.35.656.

Binkley, M., Erstad, O., Herman, J., Raizen, S., Ripley, M., Miller, M., y Rumble, M. (2012). Defining twenty-first century skills. En Assessment and teaching of 21st century skills (pp. 17-66). Países Bajos: Springer. Recuperado de: http://www.springerlink.com/ index/10.1007/978-94-007-2324-5_2.

Bordas, J., y Arras, A. (2018). Perspectivas de los estudiantes mexicanos sobre competencias en TIC, definidas por género. Revista Latina de Comunicación Social, (73), 462-477. https://doi.org/10.4185/RLCS-2018-1265.

Cabezas, M., Casillas, S., Sanches, M., y Teixeira, F. (2017). $¿$ Condicionan el género y la edad el nivel de compe- 
tencia digital? Un estudio con estudiantes universitarios. Fonseca, Journal of Communication, (15), 109-125. https://doi.org/10.14201/fjc201715109125.

Castellanos, A., Sánchez, C., y Calderero, J. (2017). Nuevos modelos tecnopedagógicos. Competencia digital de los alumnos universitarios. Revista Electrónica de Investigación Educativa, 19(1), 1-9. https://doi. org/10.24320/redie.2017.19.1.1148.

Ceneval [Centro Nacional de Evaluación para la Educación Superior] (2016). Informe anual de resultados 2015. Examen General para el Egresado de la Licenciatura en Ciencias de la Comunicación.

COSUDE [Agencia Suiza para el Desarrollo y la Cooperación] (2014). Comunicación para el desarrollo. Una guía práctica. Berna, Suiza: COSUDE.

Ferrés, J., y Piscitelli, A. (2012). La competencia mediática: Propuesta articulada de dimensiones e indicadores. Comunicar, 19(38), 75-82. https://doi.org/10.3916/ C38-2012-02-08.

García, R., Gozálvez, V., y Aguaded, J. (2014). La competencia mediática como reto para la educomunicación: instrumentos de evaluación. Cuadernosinfo, (35), 15-27. https://doi.org/10.7764/cdi.35.623.

González, N. (2012). Alfabetización para una cultura social, digital, mediática y en red. Revista Española de Documentación Cientifica, (35, n. monográfico), 17-45. https://doi.org/10.3989/redc.2012.mono.976.

González, S., y Triviño, M. (2018). Las estrategias didácticas en la práctica docente universitaria. Profesorado, Revista de Currículum y Formación del Profesorado, 22(2), 371-388. https://doi.org/10.30827/profesorado. v22i2.7728.

Grijalva, A., y Lara, J. (2019). Competencias mediáticas en jóvenes universitarios. Análisis de saberes para producir contenido digital en una IES mexicana. Edutec. Revista Electrónica de Tecnología Educativa, (67), 16-30. https://doi.org/10.21556/edutec.2019.67.1297.

Grijalva, A., y Moreno, D. (2016). Competencia mediática en jóvenes universitarios: análisis socioeducativo del currículum escolar. Sociología y Tecnociencia, 6(1), 14-25.

Grijalva, A., y Urrea, M. (2017). Competencia digitaly mediática. Panorama socioeducativo del campo en México. Ponencia presentada en Congreso Nacional de Investigación Educativa - COMIE. San Luis Potosí, México. Recu- perado de: https://www.comie.org.mx/congreso/ memoriaelectronica/v14/doc/2732.pdf.

Gumucio, A. (2011). Comunicación para el cambio social: clave del desarrollo participativo. Signo y Pensamiento, (58), 26-39.

Gutiérrez, A., y Tyner, K. (2012). Educación para los medios, alfabetización mediática y competencia digital. Comunicar, 19(38), 31-39. https://doi.org/10.3916/ C38-2012-02-03.

Latour, B. (2008). Reensamblar lo social: una introducción a la teoría del actor-red. Editorial Manantial.

Mardones, R., y Velásquez, F. (2015). Fortalecimiento de la participación comunitaria a través de la radio local: una propuesta de investigación-acción participativa (IAP) con jóvenes en Chaitén. Magallania (Punta Arenas), 43(3), 77-90. https://doi.org/10.4067/S071822442015000300007.

Martín-Barbero, J. (2011). Los oficios del comunicador. Signo y Pensamiento, 31(59), 18-40.

Ollé, C., y Cerezuela, B. (2018). Gestión de proyectos paso a paso. Editorial UOC.

Pasquali, A. (2007). Comprender la comunicación. Gedisa.

Pérez, M., y Delgado, Á. (2012). De la competencia digital y audiovisual a la competencia mediática: dimensiones e indicadores. Comunicar, 20(39), 25-34. https://doi. org/10.3916/C39-2012-02-02.

Prasad, S., Raj, D., y Bagale, S. (2017). Developing 21st century skill through project-based learning in EFL context: Challenges and opportunities. The Online Journal of New Horizons in Education, 7(1), 47-52.

Rodríguez, E. (2009). Estadística: medición, descripción e inferencia. Perspectivas Psicológicas, 6-7, 172-178. recuperado de: http://pepsic.bvsalud.org/pdf/pp/ v6e7n10/a23.pdf.

Rodríguez, R. (2020). La enseñanza de la comunicación para el cambio social en la formación de estudiantes de comunicación social y audivisual. Commons. Revista de Comunicación y Ciudadania Digital, 9(1), 26-58. https:/ / doi.org/10.25267/COMMONS.2020.v9.i1.2.

Romero-Rodríguez, L., y Aguaded, I. (2016). Consumo informativo y competencias digitales de estudiantes de periodismo de Colombia, Perú y Venezuela. Convergencia Revista de Ciencias Sociales, (70), 35-57. https:// doi.org/10.29101/crcs.v23i70.3806. 
Scott, C. (2015a). El futuro del aprendiraje 2. ¿Qué tipo de aprendizaje se necesita en el siglo XXI? UNESCO. Recuperado de: https://unesdoc.unesco.org/ark:/48223/ pf0000242996_spa.

Scott, C. (2015b). El futuro del aprendizaje 3. ¿Qué tipo de pedagogía se necesita para el siglo XXI? Investigación y prospectiva en educación. París: UNESCO.

Singh, J., Grizzle, A., Yee, S., y Culver, S. (2015). Media and information literacy for the sustainable development goals. Nordicom.

Soep, E. (2012). Generación y recreación de contenidos digitales por los jóvenes: implicaciones para la alfabetización mediática. Comunicar, 19(38), 93-100. https:// doi.org/10.3916/C38-2012-02-10.

Toro, G. (2015). Construcción de un modelo de comunicación para el desarrollo y la paz en el oriente antioqueño (Colombia) [Tesis de grado]. Universidad de Granada. Recuperado de: https://dialnet.unirioja.es/servlet/tesis?codigo $=57411$.

Tufte, T. (2015). Comunicación para el cambio social. La participación y el empoderamiento como base para el desarrollo mundial. Icaria.

Tufte, T., y Mefalopulos, P. (2009). Participatory communication: A practical guide. The World Bank. https://doi. org/10.1596/978-0-8213-8008-6.

UNESCO [Organización de las Naciones Unidas para la Educación, la Ciencia y la Cultura] (2009). Mapa de los centros y programas de formación de comunicadores y periodistas en América Latina y el Caribe (p. 146).
UNESCO (2011). Alfabetización mediática e informacional: currículum para profesores. UNESCO.

UNESCO (2013). Model curricula for journalism education: A compendium of a new syllabi. UNESCO.

Valdivia, A., Herrera, M., y Guerrero, M. (2015). Aprendizaje y producción mediática digital en la escuela: un abordaje etnográfico del aprendizaje como práctica cultural en artes visuales. Estudios Pedagógicos, (41, especial), 231-251. https://doi. org/10.4067/S0718-07052015000300015.

Van Laar, E., Van Deursen, A., Van Dijk, J., y de Haan, J. (2020). Determinants of 21st-century skills and 21st-century digital skills for workers: A systematic literature review. SAGE Open, 10(1), 1-14. https:// doi.org/10.1177/2158244019900176.

Wiek, A., Bernstein, M., Foley, R., Cohen, M., Forrest, N., Kuzdas, C., Kay, B., y Keeler, L. (2016). Operationalising competencies in higher education for sustainable development. En Routledge Handbook of Higher Education for Sustainable Development (pp. 241-260). Routledge.

Zermeño, A., Padilla de la Torre, R., y Fernández, A. (2020). Formación de comunicadores para el desarrollo y el cambio social. Análisis de experiencias en el centro-occidente de México. Commons. Revista de Comunicación y Ciudadanía Digital, 9(1), 59-88. http:// doi.org/10.25267/COMMONS.2020. v9.11.3.

Cómo citar este artículo:

Navarrete Vega, M. A., y Romero González, R. M. (2021). Estrategia didáctica para fortalecer las competencias de producción mediática desde la comunicación para el desarrollo. IE Revista de Investigación Educativa de la REDIECH, 12, e1208. doi: 10.33010/ie_rie_rediech.v12i0.1208. 